\title{
Preparing for the Next Normal: Transformation in the Role of Medical Affairs Following the COVID-19 Pandemic
}

\author{
Romik Ghosh $^{1} \cdot$ Senthilnathan Mohanasundaram ${ }^{1} \cdot$ Sujatha Shetty ${ }^{1} \cdot$ Shalini Menon $^{1}$
}

Accepted: 9 June 2021 / Published online: 5 July 2021

(c) The Author(s) 2021

\begin{abstract}
The medical affairs function represents one of the scientific interfaces in a pharmaceutical organization. Over the last two decades, medical affairs has evolved from being a support function to a strategic pillar within organizational business units. The COVID-19 pandemic has given rise to unforeseen circumstances resulting in a dramatic change in external stakeholder engagements, catapulting the medical affairs function into leading the way on scientific engagements and patient-centric endeavors. The changes in stakeholder interactions and behavior as a result of the pandemic last year are likely to persist in the foreseeable future for which medical affairs professionals need to enhance existing skill sets and acquire expertise in newer domains. In this paper, the transformation of the medical affairs team to a key strategic partner and the skills required to strengthen this transition, in the next normal of a post-COVID world, is explored.
\end{abstract}

\section{Introduction}

The medical affairs (MA) function is one of the powerhouses of scientific expertise in a pharmaceutical organization. Traditionally, the MA function was tasked with providing scientific support by communicating medical information to healthcare professionals (HCPs). On the commercial front, MA was involved in reviewing and approving promotional messages and materials to conform to the evidence, guidelines, and regulations. Other responsibilities included training commercial teams to improve their understanding of products and therapy areas, preparing content for educational programs, facilitating clinical trials, and supporting investigator-initiated studies [1].

The increasing complexities of drug development, a greater emphasis on individualization of treatment, stricter laws, and regulations, an increased scrutiny on the interactions between pharmaceutical organizations and HCPs, and rapidly changing expectations of different stakeholders have driven the transformation of the MA function into an important strategic partner [2]. Further, the COVID-19 pandemic led to a drastic change such as a decrease of faceto-face interactions and educational programs; a decrease in accessibility to HCPs and a slowing down of clinical trials and related publications. The ongoing pandemic is changing

Senthilnathan Mohanasundaram

Senthilnathan.Mohanasundaram@sanofi.com

1 Medical Affairs, Sanofi India Limited, Mumbai, India

\section{Key Points}

The restrictions in movement and access brought about by the COVID-19 pandemic have triggered an alteration in the engagement models with stakeholders of the pharmaceutical industry including the healthcare professionals (HCPs).

Medical affairs (MA) professionals have felt this impact and need to recalibrate their role and acquire a new set of competencies to stay abreast with the rapidly changing environment.

This was further substantiated by a first of its kind HCP survey indicating a paradigm shift in their preference for virtual scientific interactions.

To be future-ready, MA professionals need exponential skill enhancements like adopting omni-channel engagement, fostering external partnerships, evidence generation in real-world settings and radical skill advancements like upgrading technological knowledge and inculcating a patient-centric focus with a "beyond the pill" mindset. Periodic assessments of MA initiatives in terms of format, channel, content, duration, and relevance to clinical practice could be integrated into the performance metrics. 
the way pharmaceutical organizations function, in terms of customer interactions and scientific engagements. It has triggered a sudden surge of digital interactions via hitherto relatively unexplored channels, leading to logistic and technical challenges. This has led to an acceleration in the evolution of MA as one of the three strategic pillars in the pharmaceutical organization along with research and development, and commercial plus market access functions [1-3]. The ongoing situation has disrupted numerous working models in the industry; and many of the changes will continue to persist and redefine patient and physician experiences in the postpandemic era as well. The changing mindset of the HCPs and the way they would like to interact with MA teams in the foreseeable future is elucidated in our survey, which sought to assess feedback on the medical initiatives undertaken during the pandemic.

To keep pace with the demands of the changing times, MA professionals must acquire a whole new set of competencies. In this paper, the transformation of the MA teams and the skills required for a smooth transition in the next normal are explored.

\section{Transformation of MA Function in the COVID Era}

The immediate impact after the onset of COVID-19 pandemic in India and the subsequent nationwide lockdown between March and June 2020 could not be foreseen and MA teams had to overcome multiple challenges to adapt to the changing environment and cater to the enormous demand for clinically meaningful information regarding COVID-19. As also observed in other parts of the world, the immediate consequences at the onset of the pandemic and the enforcement of a lockdown were as follows [3, 4].

Decreased access to HCPs and institutions:

- Face-to-face interactions with HCPs and medical education programs were abruptly halted.

- Congresses (international and national) were either canceled or hastily shifted to virtual platforms, further limiting face-to-face scientific exchanges for abstracts, posters, and oral presentations as well as medical booth interactions.

- As a result, opportunities to network with HCPs and gather insights were adversely impacted.

- Clinical trials slowed down because of accessibility issues.

- Planned publications of studies were also affected leading to valuable information being unavailable within expected timelines.
Rise in virtual engagements: This was the direct result of decreased physical access to external stakeholders. The challenges encountered included adapting to newer alternate channels by HCPs and inadequate network connectivity.

Need for information beyond brands: As COVID-19 impacted multiple organ systems, HCPs who were often focused only on their areas of specialty had to focus on the overall management and impact of the novel coronavirus on their respective specialty; hence they needed information beyond the class of medications, brands, and therapy areas.

The shifts made by the MA function to address these changed dynamics were:

- Fulfilling the instantaneous need in raising awareness, dispelling misinformation, and enhancing knowledge amongst HCPs on the rapidly evolving COVID-19 situation.

- Shifting the scientific content strategy from a brand focus to an emphasis on COVID-19 and its relation to co-morbidities, disease states, and therapeutic areas of specialization of the HCPs.

- Adapting to new means of connecting to customers by going completely virtual to ensure timely dissemination and a wider reach.

\subsection{Feedback of HCPs on the Transformation Brought About by MA}

While the MA teams adapted to the changing environment rapidly, HCPs across India also molded to the new means of interaction with pharmaceutical organizations. According to a recent market research report from M3, HCPs cited "updated information" to be the key for curbing the COVID19 spread and reached out to MA to obtain reliable evidencebased information for managing their patients [3].

Taking a cue from this finding, an online pan-India survey was conducted by the Sanofi medical team (see Electronic Supplementary Material for the survey questionnaire). The objective of the survey was to understand the HCP requirements and feedback in the evolving environment. The HCP survey, conducted between 8 December, 2020 and 25 February, 2021, involved 191 respondents from various therapy areas. The survey sought response on the delivery formats, channels, type of content, and relevance of the same to clinical practice. General updates and information related to managing COVID-19 based on specialty was the most sought-after information. The survey also found that $93 \%$ of the HCPs appreciated the innovative formats of information exchange during the pandemic. 


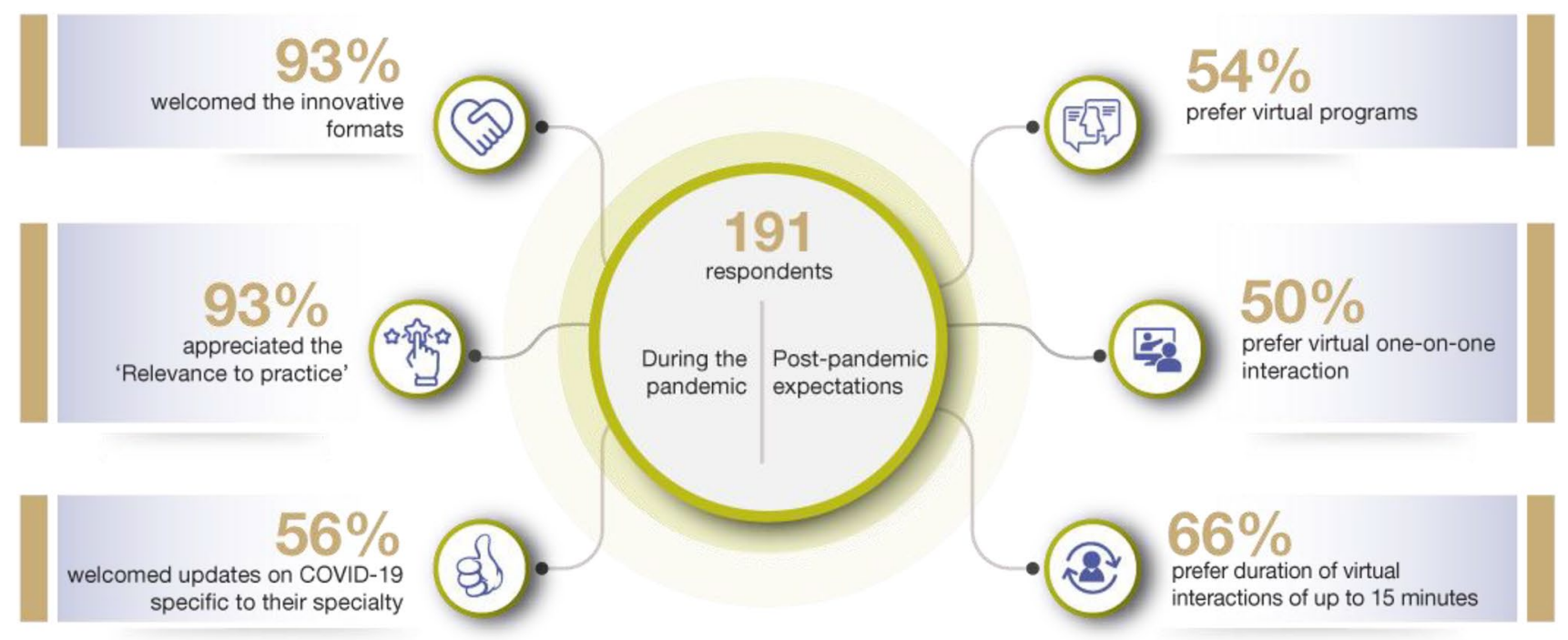

Fig. 1 Findings from the healthcare professional survey

One of the components of this survey was to elicit feedback on the virtual channels of scientific data dissemination used by MA during the COVID-19 pandemic. Amongst virtual channels that included telecommunication messages, e-mails, one-to-one interactions, educational programs with a limited number of participants, and large-scale scientific events, $51 \%$ of the HCPs preferred educational programs involving fewer than 20 participants.

Most HCPs indicated a preference to have a mix of virtual and in-person engagements even after the pandemicenforced restrictions are relaxed and regular face-to-face interactions resume. Interestingly, as per this survey, $54 \%$ of the respondents indicated a preference for a higher proportion of virtual programs compared to face-to-face programs. Additionally, for the one-on-one interactions, $50 \%$ of the respondents opted for a higher proportion of virtual channel over in-person meetings. The majority of the HCPs (66\%) considered the optimal duration of a virtual interaction as 15 min (Fig. 1).

The findings of this survey provided direction to the MA team to understand the requirements of the HCPs during the pandemic and re-align their focus where applicable. The survey results may also help MA teams across the industry to recalibrate their future strategies. These periodic assessments of MA initiatives in terms of format, channel, type of content, duration, and relevance to clinical practice could also be integrated into the performance metrics. Further large-scale and in-depth surveys may throw more light on the requirements of HCPs and help assess the impact of the educational initiatives adopted by MA.

\section{Preparing for the Next Normal}

The key learnings from the changes in the working environment during the pandemic that will help MA in the future include:

- Anticipating and addressing the changing information needs of HCPs in real time [5].

- Gaining expertise in areas beyond therapy such as evolving guidelines, government regulations, and recommendations of global and local associations.

- Priming and equipping the medical information department for the sudden spike in the need for scientific information and inquiries.

- Reducing the "global to local" turn-around time with agile cross-functional and cross-affiliate collaborations.

- Leveraging multi-channel engagement models and at the same time overcoming webinar and digital fatigue by introducing differentiating and interactive features such as polls and break-out sessions [6,7].

- Exploring sources of agile data generation by using realworld analytics $[8,9]$.

Apart from the key learnings, MA teams would need to hone specific skills and embrace innovation to be capable to meet the expectations of various stakeholders. These capability building attributes would require an exponential enhancement in the existing skill set, while a few of them would require a radical shift in the approach of working (Fig. 2). 


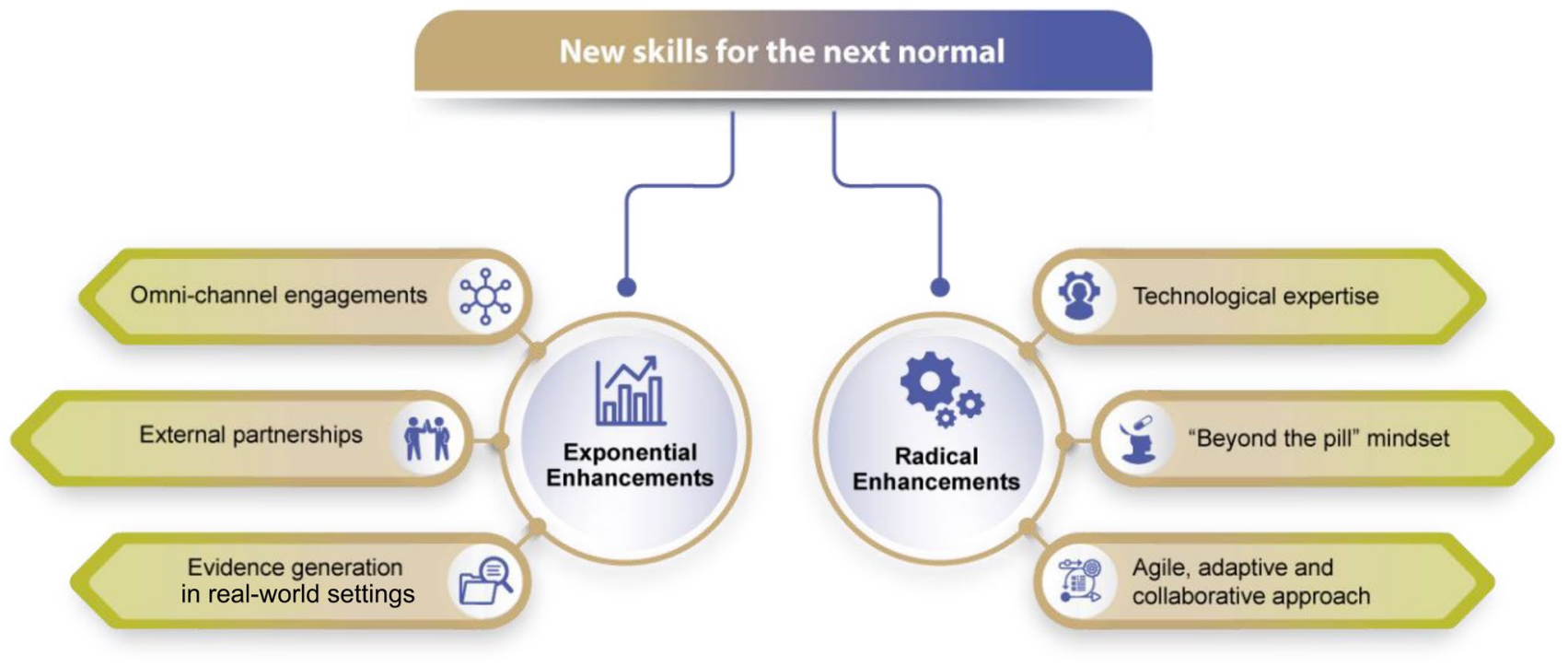

Fig. 2 Desired medical affairs skill set for the next normal

\subsection{Exponential Enhancement of Existing Skill Set}

\subsubsection{Adopting Omni-Channel Engagement by Building on a Digital Event Backbone}

Medical education programs conducted virtually will continue to play an important role in knowledge dissemination and enhancement for HCPs. Medical affairs teams should direct their efforts to enhance this digital experience by exploring opportunities beyond didactic webinars such as:

- Group discussions with peers.

- Access to medical information in the webinar platforms.

- Access to accreditation programs and certifications.

- Online quiz, contests, and gamification.

The challenges towards achieving this experiential outcome could be related to inadequate connectivity, complexity in navigating the deployed platform, and content overload, which needs special attention and course correction, if required.

\subsubsection{External Partnerships}

The pandemic stimulated several organizations to collaborate to respond to the drastically changing environment. In the new normal, this also must be pursued. Some of the areas where the industry can work together are co-opting the service model along with the product-based model, co-organizing medical awareness/education programs, and deployment of data platforms and in capability building exercises within the pharmaceutical physician community. Medical affairs can be an instrumental force in managing and developing such partnerships with other organizations in line with the internal compliance and legal frameworks.

\subsubsection{Evidence Generation in Real-World Settings}

At the onset of the pandemic, many MA organizations had to instantly support real-word evidence generation studies and investigator-initiated studies in patients with COVID. In the post-COVID-19 scenario, the emphasis on real-world evidence and investigator-initiated studies is likely to persist and amplify. Medical affairs will need to be familiar with multiple data platforms, means of collecting real-world data (which includes electronic health records), connected systems including wearables, regulations around real-world studies, designing, analyzing, and interpreting results, and effective communication of real-world evidence.

\subsection{Radical Enhancement of Existing Skill Set}

Further to the exponential enhancements outlined above, there is a need to radically build upon the following competencies.

\subsubsection{Technological Expertise [9]}

This is a growing requirement for the MA professionals in the future as technological evolution is driving the transformation in treatment patterns and HCP behaviors. Apart from using digital channels for scientific communication, it involves understanding the application of artificial intelligence, machine learning, and big data analytics. 


\subsubsection{Beyond the Pill Mindset}

With a growing emphasis of patient centricity, MA teams need to think beyond the product and embrace the service model that would augment the product and ensure optimal outcomes for the patients. Medical affairs team should be subject matter experts of the medical offerings that will come under the scope of the service model and consider developing content around the same.

\subsubsection{Agility, Adaptability, Collaboration [8]}

To be successful in the current scenario, which brings with it changes in the system of working and new expectations from HCPs, MA teams will have to be agile, quick in adapting to the changing working environment, embrace crossfunctional collaborations, be apprised of developments in the external environment, assess the impact of situations, and provide new approaches and ideas to improve the quality of care. They will also need to focus on business leadership and strategic vision coupled with a need to assimilate the current learnings and adapt these to future use both within and outside the core medical domain.

\section{Conclusions}

Medical affairs is on the cusp of a unique opportunity to transform the pharmaceutical industry's future, with the unprecedented changes generated by the COVID-19 pandemic. Medical affairs must seize this opportunity to embrace a customer-centric approach and focus on crossfunctional and external collaborations to implement technological changes and deliver excellence in science. Medical affairs needs to intensify focus on omni-channel scientific engagement, explore possibilities of external partnerships, and prioritize evidence-generation projects. The deep-rooted medical expertise within MA teams needs to be augmented by several radical shifts in the working approach. To make this happen, equal emphasis should be placed on self-development within the MA teams to equip them to handle the evolving requirements of the future. Medical affairs teams of tomorrow would have to be technologically agile, rapidly adapt to the changing healthcare environment, fulfill multi-stakeholder needs with a patient-centric focus, and look "beyond the pill" to stay competitive and drive the scientific growth engine of the pharmaceutical industry in a post-pandemic world.

Supplementary Information The online version contains supplementary material available at https://doi.org/10.1007/s40290-021-00392-x.

\section{Declarations}

Funding Medical writing and open access-related charges were paid by Sanofi India. We acknowledge Rajshri Mallabadi and Poornima Iyer from BioQuest Solutions Pvt. Ltd, Bangalore, for providing medical writing and editorial support in the preparation of this manuscript, which was paid for by Sanofi, India.

Conflict of Interest Romik Ghosh, Senthilnathan Mohanasundaram, Sujatha Shetty, and Shalini Menon are permanent employees of Sanofi India. The opinions expressed in this article are those of the authors and not necessarily those of Sanofi India.

Ethics Approval Not applicable.

Consent to Participate Not applicable.

Consent for Publication Not applicable.

Availability of Data and Material Not applicable.

Code Availability Not applicable.

Author Contributions All authors have reviewed the paper and then discussed within the author team to arrive at a final version. All authors have read and approved the final version of the submitted paper.

Open Access This article is licensed under a Creative Commons Attribution-NonCommercial 4.0 International License, which permits any non-commercial use, sharing, adaptation, distribution and reproduction in any medium or format, as long as you give appropriate credit to the original author(s) and the source, provide a link to the Creative Commons licence, and indicate if changes were made. The images or other third party material in this article are included in the article's Creative Commons licence, unless indicated otherwise in a credit line to the material. If material is not included in the article's Creative Commons licence and your intended use is not permitted by statutory regulation or exceeds the permitted use, you will need to obtain permission directly from the copyright holder. To view a copy of this licence, visit http://creativecommons.org/licenses/by-nc/4.0/.

\section{References}

1. Evers M, Ghatak A, Suresh B. McKinsey \& Company: a vision for medical affairs in 2025. 2019. https://www.mckinsey.com/indus tries/pharmaceuticals-and-medical-products/our-insights/a-visionfor-medical-affairs-in-2025. Accessed 1 Mar 2021.

2. Paardekooper C. Medical affairs in transition, towards a fully integrated model. Vintura. 2019. https://www.vintura.com/en/life-scien ce-consulting/publications/whitepaper-medical-affairs-in-transitiontowards-a-fully-integrated-model/. Accessed 1 Mar 2021.

3. Mckinsey and Company. COVID-19 implications for life sciences R\&D: recovery and the next normal. 2021. https://www.mckinsey. com/industries/pharmaceuticals-and-medical-products/our-insig hts/covid-19-implications-for-life-sciences-r-and-d-recovery-andthe-next-normal\#. Accessed 1 Mar 2021.

4. Rajadhyaksha VD. Medical affairs post-COVID 19: are we ready to take the baton? Perspect Clin Res. 2020;11:124-7 (PMID: 33033702; PMCID: PMC7513787).

5. van Tongeren $\mathrm{T}$. The state of customer experience in the pharmaceutical industry, 2018: HCP interactions. April 2019. 2019. 
https://dtconsulting.com/the-state-of-customer-experience-in-thepharmaceutical-industry-2018-hcp-interactions. Accessed 1 Mar 2021.

6. John S. Addressing COVID-19 challenges: quick wins for medical affairs leaders. 2021. https://www.zs.com/insights/addre ssing-covid-19-challenges-quick-wins-for-medical-affairs-leade rs. Accessed 1 Mar 2021.

7. Desai A. ViewPoints article: digital revolution in healthcare and strategic role of medical affairs amidst Covid-19 outbreak. 2021. https://pharmashots.com/36266/insights-kol-articles-digital-revol ution-in-healthcare-and-strategic-role-of-medical-affairs-amidstcovid-19. Accessed 1 Mar 2021.

8. Bedenkov A, Rajadhyaksha V, Beekman M, et al. Developing medical affairs leaders who create the future. Pharmaceut Med. 2020;34(5):301-7. https://doi.org/10.1007/s40290-020-00351-y (PMID:32949352;PMCID:PMC7501759).

9. Bedenkov A, Moreno C, Agustin L, et al. Customer centricity in medical affairs needs human-centric artificial intelligence. Pharmaceut Med. 2021;35(1):21-9. https://doi.org/10.1007/s40290020-00378-1 (PMID:33464482;PMCID:PMC7814264). 